\title{
COVID-19 and cancer: do we really know what we think we know?
}

Andrew G. Robinson ${ }^{1,2 凶}$, Bishal Gyawali $^{1,2}$ and Gerald Evans (10 ${ }^{3}$

Early published data on COVID-19 in patients with cancer are being referenced in clinical guidelines, despite methodological flaws that limit the quality of much of this evidence. In the next phase of research in this area, we argue that the quality of observational evidence should be prioritized over speed of publication.

The past few months have seen an explosion of published research and guidelines regarding cancer care in the context of the coronavirus disease (COVID-19) pandemic. Unsurprisingly, many authors and commentators have suggested that some cancer therapies - particularly those with immunosuppressive effects - should be delayed or reconsidered based on the presumption that patients with cancer have a higher risk of contracting COVID-19 and/or having worse outcomes. However, owing to the limited level of evidence, it is important to reflect on our practices and test these presumptions as more and/or stronger evidence accumulates. In this commentary, we provide a narrative synthesis of studies on how COVID-19 can affect patients with cancer and highlight various methodological pitfalls that might limit the reliability of these data (Supplementary TABLE 1). Oncologists need to avoid making decisions based on poor-quality evidence ${ }^{1}$.

\section{Risks of SARS-CoV-2 infection}

To date, the literature on this topic has been challenged by the very definition of what it means to have cancer'. Among various case series, the proportion of non-selected patients with COVID-19 and cancer ranges from $0.5 \%^{2}$ to $6 \%^{3}$, thus illustrating the issue of which cancer cases are counted. In an analysis of data from a cohort of 1,590 patients with COVID-19, the authors reported data from 18 patients ( $1.13 \%$ of the cohort) with a history of cancer ${ }^{4}$, noting that this percentage is substantially higher than that of the general population $(0.29 \%)$. This appears to be a comparison of an incidence rate $(0.29 \%)$ with a prevalence rate $(1.13 \%)$. In examining the appendix of this report ${ }^{4} 6$ of the 18 patients with cancer were diagnosed with cancer $<2$ years before being diagnosed with COVID-19, while the other 12 had been diagnosed a median of 4.5 years previously. Elsewhere, Yu et al. ${ }^{5}$ reported that 12 of 1,524 patients presenting with COVID-19 symptoms had a history of cancer $(0.79 \%)$, although only four of these patients had laboratory confirmed COVID-19 (defined as being severe acute respiratory syndrome coronavirus 2
(SARS-CoV-2)-positive by PCR, in addition to previous contact with infected individuals, symptoms and/or ground-glass opacities on chest CT).

In a presentation of data from Gustave Roussy Cancer Centre, France ${ }^{6}$, the authors reported the number of patients with cancer who were either inpatients or had outpatient appointments between 14 March and 15 April $(7,251)$, were tested for SARS-CoV-2 $(1,302)$ and who tested positive for SARS-CoV-2 (156), indicating an infection rate of $2.1 \%$, or $12 \%$ of those tested. The proportion of patients tested (18\%) and infection rate $(2.1 \%)$ seem higher than that of the broader population of France, with a $0.71 \%$ test rate, with $25 \%$ testing positive resulting in a $0.25 \%$ infection rate ${ }^{6}$. Thus, based on current evidence, patients with cancer might plausibly have a higher risk of acquiring SARS-CoV-2 infections. Comparisons of the prevalence of cancer among patients with COVID-19 to that of cancer in the community should be clear to use a similar definition of prevalence. Investigators comparing the incidence of COVID-19 among patients with cancer to that of a control group should provide clear definitions of testing criteria and cases in order to determine the extent to which the results are limited by ascertainment bias.

\section{Risks of adverse COVID-19 outcomes}

An important question remains unanswered: are patients with cancer more likely to die from COVID-19? To answer this question, we must know if patients with cancer and COVID-19 are more likely to die from COVID-19, rather than with COVID-19, compared with those without cancer. Reported case-fatality rates (CFRs) range from $5.6 \%$ in the large series by Wu et al. ${ }^{2}$ $(n=107)$ to $29 \%$ in the smaller series by Zhang et al. $(n=28)$. CFR is dependent on what constitutes a fatality (whether a fatality is attributed to COVID-19 or cancer) and on what constitutes a case of COVID-19.

Dai et $\mathrm{al}^{8}{ }^{8}$ described the outcomes of 105 patents with COVID-19 and cancer and compared them to a control group of 536 patients with COVID-19 who were matched for age, sex and comorbidities. The percentage 
Researchers must ensure that our policy-makers have the highestquality evidence upon which to make potentially monumental decisions of patients with laboratory confirmed COVID-19 was not provided. The majority of patients (54\%) were diagnosed with cancer within the previous year, and $16 \%$ had metastatic disease. The death rate and complication rates were significantly higher for patients with cancer (OR for death 2.34, 95\% CI 1.15-4.77; $P=0.03$ ). After adjusting for age, smoking status and comorbidities, the OR for death was $2.17(P=0.06)$. The risks of death or serious and/or critical symptoms appears to be mainly driven by the $16 \%$ of patients with metastatic disease (OR 5.58, 95\% CI 1.7-18.23; $P=0.01$ and OR 5.97, 95\% CI 2.24-15.91; $P<0.01$, respectively). These data suggest that the reported risks of poor outcomes reflect either residual confounding (for example, because patients with end-stage cancer might be more likely to die from COVID-19 because they have inherently worse general health and might already have compromised organ function) or misattribution of deaths.

Data from another study presented in April 2020 indicate a CFR of $34 \%(66 / 191)^{9}$ in patients with thoracic cancer (75\% with non-small-cell lung cancer) diagnosed with COVID-19 and included both laboratory confirmed and clinically defined disease. Distinguishing the death rates among patients with clinically defined COVID-19 versus those with laboratory-confirmed disease is important given the potential for misclassification of patients with complex changes in the lungs and the fact that infectious pulmonary complications other than COVID-19 often contribute to the terminal event in patients with lung cancer. Among seven patients with lung cancer described by Yu et al. ${ }^{5}$, only one had laboratory confirmed COVID-19.

Data from Gustave Roussy Cancer Centre ${ }^{6}$ indicate a CFR of $15 \%$ (20/137), which appears comparable to the $17 \%$ CFR in Paris during the same time period. However, the likelihood of SARS-CoV-2 testing appears much greater in patients with cancer, and this increases the likelihood of detecting asymptomatic and/or mildly symptomatic cases of COVID-19 in this population, which would skew the CFR in favour of those with cancer. Nonetheless, one other report also failed to detect a higher CFR in patients with cancer ${ }^{3}$.

Data from Italy highlight the issue of attribution of death (distinguishing patients who die with COVID-19 from those who die from COVID-19). These investigators report that approximately $16 \%$ of patients who died with or from COVID-19 had 'active cancer' ${ }^{10}$. The reported practice in Italy was to consider COVID-19 the cause of death of all patients who died with the disease, and CFRs for patients with cancer may be inflated.

Patients with a history of cancer who develop COVID-19 might have a higher risk of death from COVID-19 than those without cancer, although the evidence to date does not provide a definitive answer. If a true difference exists, whether this is driven by a more severe form of viral infection versus overlapping risk factors for both COVID-19 fatality and cancer (such as age, frailty, smoking history, obesity and organ dysfunction) versus simply reduced physiological reserves, remains unclear. CFRs are subject to biases - both in the definition of a case and the attribution of fatality, and CFRs should be reported separately for those with laboratory confirmed versus clinically defined COVID-19.

\section{Effects of specific treatment modalities}

Evidence for the effects of specific treatment modalities on COVID-19 outcomes is, thus far, inconclusive. Data published by Liang et al. ${ }^{4}$ have been extensively cited as evidence of an increased risk, although these conclusions are based on data from only four patients who received treatment in the previous month: one with an adrenalectomy, one with immunotherapy for colorectal cancer and two with cytotoxic chemotherapy for lung cancer.

In the report by Zhang et al. ${ }^{7}(n=28)$, the majority of patients (five of six) who received therapy $\leq 14$ days before diagnosis of COVID-19 had severe COVID-19 symptoms, compared with 10 of 22 who received treatment $>14$ days prior to a COVID-19 diagnosis. These observations led the investigators to conclude that treatment within 14 days is a risk factor for adverse COVID-19 outcomes $^{7}$. However, when the cutoff is extended to 30 days, this effect appears to reverse, with 5/12 patients (42\%) who received treatment within 30 days prior to a COVID-19 diagnosis having severe COVID-19 symptoms compared with 10 of $16(63 \%)$ in those treated $>30$ days previously. Given this apparent reversal of 'effect' when the cutoff point is adjusted, it seems possible that the association reported at 14 days is an artefact driven by small numbers.

Adequately powered reports of complication rates or death rates by treatment modality received are needed in order to determine if treatment adjustments are necessary and to determine how long a patient can be deemed to be at a higher risk. The rationale for time-related cutoffs should be clear and based on what is known about a specific cancer therapy (such as the dose and/or duration). Based on current knowledge, insufficient evidence currently exists to recommend that one (or more) forms of cancer therapy should be avoided or delayed in the context of COVID-19.

\section{Conclusions}

While having cancer and receiving certain cancer therapies remain plausible risk factors for both contracting SARSCoV-2 infections and having more severe COVID-19 outcomes, existing data do not yet answer these questions. Notably, the early publications in this area include data from a very small number of patients, but have nonetheless had substantial effects. The extensively cited study by Liang et al. ${ }^{4}$ is often used as justification for 'a possible increased risk' associated with chemotherapy, despite only two patients in this analysis receiving systemic chemotherapy within the month before COVID-19 diagnosis. Data from Yu et al. ${ }^{5}$ have been used to support universal screening of patients with lung cancer for SARS-CoV-2 infections, even though this cohort contained only seven patients with lung cancer, six of whom did not have laboratory-confirmed COVID-19. It is difficult to imagine any other context in which data from such small, highly selected, and often flawed case series would be published in major journals and have such a substantial influence on clinical practice and policy.

The collective research output from the oncology community is testament to the commitment of 
our profession in light of a very challenging context. However, we need to ensure that the data are viewed critically and with the same level of rigour as we apply outside the context of a viral pandemic. Researchers must ensure that our policy-makers have the highest-quality evidence upon which to make potentially monumental decisions. As eloquently stated in a commentary by London and Kimmelman published in April 2020 "Crises are no excuse for lowering scientific standards" This quote is true both of clinical trials and of observational evidence. The descriptive data on cancer and COVID-19 discussed in this commentary might all be subject to high levels of bias, which could have unintended consequences by unduly influencing physicians' practice or clinical guidelines, particularly if conclusions are cited and propagated without either context or the acknowledgement of high levels of uncertainty.

1. London, A. J. \& Kimmelman, J. Against pandemic research exceptionalism. Science 368, 476-477 (2020).

2. Wu, Z. \& McGoogan, J. M. Characteristics of and important lessons from the coronavirus disease 2019 (COVID-19) outbreak in China: summary of a report of 72314 cases from the Chinese Center for Disease Control and Prevention. JAMA 323, 1239-1242 (2020).

3. Miyashita, H. et al. Do patients with cancer have a poorer prognosis of COVID-19? An experience in New York City. Ann. Oncol. https:// doi.org/10.1016/j.annonc.2020.04.006 (2020).
4. Liang, W. et al. Cancer patients in SARS-CoV-2 infection: a nationwide analysis in China. Lancet Oncol. 21, 335-337 (2020).

5. Yu, J., Ouyang, W., Chua, M. L. K. \& Xie, C. SARS-CoV-2 transmission in patients with cancer at a tertiary care hospital in Wuhan, China. JAMA Oncol. https://doi.org/10.1001/jamaoncol.2020.0980 (2020).

6. Barlesi, S. F et al. Outcome of cancer patients infected with COVID-19, including toxicity of cancer treatments https://www. abstractsonline.com/pp8/\#!/9045/presentation/10935 (2020).

7. Zhang, L. et al. Clinical characteristics of COVID-19-infected cancer patients: a retrospective case study in three hospitals within Wuhan China. Ann. Oncol. https://doi.org/10.1016/j.annonc.2020.03.296 (2020).

8. Dai, M. Y. et al. Patients with cancer appear more vulnerable to SARS-CoV-2: a multi-center study during the COVID-19 outbreak. Cancer Discov. https://doi.org/10.1158/2159-8290.CD-20-0422 (2020).

9. Garissino, M. TERAVOLT (Thoracic cancERs international coVid 19 cOLlaboraTion): first results of a global collaboration to address the impact of COVID-19 in patients with thoracic malignancies. https://www.abstractsonline.com/pp8/\#!/9045/presentation/10927 (2020).

10. Trapani, D., Marra, A. \& Curigliano, G. The experience on coronavirus disease 2019 and cancer from an oncology hub institution in Milan, Lombardy Region. Eur. J. Cancer 132, 199-206 (2020).

Acknowledgements

The authors gratefully acknowledge Dr. Christopher Booth (Queen's University) for contributing to this manuscript.

Competing interests

The authors declare no competing interests.

Supplementary information

Supplementary information is available for this paper at https://doi.org/ 10.1038/s41571-020-0394-y. 